Address for Correspondence: Dr. Ling Wang, MD, PhD, 301 Yanchangzhong Road Department of Nephrology \& Rheumatology,

Shanghai Tenth People's Hospital, Tongji University School of Medicine,

Shanghai, 200072, China Email: nowax@126.com

Dr. Chandra Mohan, MD, PhD, 3605 Cullen Blvd,

Department of Biomedical Engineering,

University of Houston,

Houston, TX 77204, USA

Email: cmohan@central.uh.edu

\begin{tabular}{|l|}
\hline Access this article online \\
\hline Website: \\
www.intern-med.com \\
\hline DOI: \\
10.1515/jtim-2016-0033 \\
\hline Quick Response Code: \\
\hline \\
\end{tabular}

\title{
Contrast-enhanced ultrasound: A promising method for renal microvascular perfusion evaluation
}

\author{
Ling Wang ${ }^{1}$ Chandra Mohan ${ }^{1,2}$ \\ 'Department of Nephrology \& Rheumatology, Shanghai Tenth People's Hospital, Tongji University School of \\ Medicine, Shanghai, 200072, China; \\ ${ }^{2}$ Department of Biomedical Engineering, University of Houston, Houston, TX 77204, USA
}

\section{ABSTRACT}

This article reviews the application of contrast-enhanced ultrasound (CEUS) in gauging renal microvascular perfusion in diverse renal diseases. The unique nature of the contrast agents used in CEUS provides real-time and quantitative imaging of the vasculature. In addition to the traditional use of CEUS for evaluation of kidney masses, it also emerges as a safe and effective imaging approach to assess microvascular perfusion in diffuse renal lesions, noninvasively. Although the precise CEUS parameters that may best predict disease still warrant systematic evaluation, animal models and limited clinical trials in humans raise hopes that CEUS could outcompete competing modalities as a first-line tool for assessing renal perfusion non-invasively, even in ailments such as acute kidney injury and chronic kidney disease.

Key words: ultrasound, microvascular perfusion, acute kidney injury, chronic kidney disease, imaging, glomerular filtration rate, computed tomography, magnetic resonance imaging

\section{INTRODUCTION}

Cumulative evidence indicates that renal hemodynamic changes underlie several kidney diseases with diffuse lesions ${ }^{[1,2]}$. Hyperperfusion has been documented in progressive glomerular injury, especially in diabetic kidney disease (DKD) ${ }^{[1]}$; renal blood flow (RBF) has been found to be changed in septic acute kidney injury (AKI) ${ }^{[2,3]}$; renal dysfunction has also been associated with a significant change in cortical perfusion ${ }^{[4]}$. Contrast-enhanced computed tomography (CT) and magnetic resonance imaging (MRI) have been used to measure RBF and glomerular filtration rate ${ }^{[5]}$. However, CT and dynamic MRI techniques for measuring blood perfusion often depend on intravenous injection of contrast agents ${ }^{[6,7]}$, which are potential hazards in patients with renal insufficiency ${ }^{[8]}$. Although functional MRI can be performed without contrast agents, the invasive nature of the pharmacological or physiological manipulations associated with this approach $^{[9]}$, and the lack of standardized sequences, post-processing software and models hinder its routine application in the clinic ${ }^{[10]}$.

Contrast-enhanced ultrasound (CEUS) is a competing technology that enables realtime observation of vascular perfusion. The basic composition of the contrast agents are microbubbles made of gases embedded within a shell, which can function as red blood cell tracers. Their ability to reflect ultrasound and avoid destruction during circulation allows for continuous imaging of the vasculature and blood flow ${ }^{[11]}$. These contrast agents are well-regarded for their renal safety, high tolerance, and lack of radiation ${ }^{[12]}$. Hence, CEUS is widely used for the diagnosis of various kidney masses, such as tumors, cystic lesions, infarction, cortical necrosis, and trauma-induced lesions, among others.

However, it is now becoming evident that changes in renal perfusion may also help predict and monitor the progression of other renal diseases. CEUS can provide 
real-time, noninvasive and relative quantitative estimates of renal microvascular perfusion independent of kidney function. This review will focus on the potential applications of CEUS in monitoring renal microvascular perfusion in various kidney diseases.

\section{CONVENTIONAL APPLICATIONS OF CEUS IN NEPHROLOGY}

European Federation of Societies for Ultrasound in Medicine and Biology (EFSUMB) Guidelines and Recommendations, updated in 2011, had documented the potential indications of CEUS in kidney diseases ${ }^{[13]}$. Besides their echogenicity, renal tumors exhibit altered vascularity compared to the normal parenchyma, and CEUS can be helpful in their differential diagnosis ${ }^{[14]}$. For detection of cyst-like lesions, CEUS is even more sensitive than contrast-enhanced CT ${ }^{[15]}$. Owing to the excellent spatial resolution of CEUS, cortical necrosis is shown as a non-enhancing area with vascularity. CEUS is also superior to color Doppler ultrasound in diagnosing renal infarction, because CEUS can detect slower flow in smaller blood vessels. Kidney abscesses can also be confirmed and followed up using CEUS ${ }^{[16]}$. Since contrast agents are not concentrated in the collecting system, CEUS assessment of kidney trauma cannot rule out pelvi-calyceal and ureteric injuries; therefore, CEUS may be better suited for limited injuries, or multiple solid organ traumas ${ }^{[17]}$.

\section{RENAL MICROVASCULAR PERFUSION EVALUATION IN ANIMAL MODELS}

The application of CEUS for studying renal microvascular perfusion has been examined in many animal models. Histological assessment in porcine models have shown that the ultrasound contrast agent used (sulfur hexafluoride) did not inflict any tissue damage to the kidneys ${ }^{[18]}$. These findings suggest that CEUS could be safe for regular use in humans, with minimal risk of tissue damage. Kogan et al. placed transit-time flow probes in renal arteries of rats to measure the volume of $\mathrm{RBF}$, and compared these readouts to those reported by CEUS. They reported that CEUS-derived parameters were comparable to absolute measurements of blood flow in rat kidneys $(\mathrm{R}>0.9)^{[19]}$. Their results demonstrated that CEUS could indeed reflect RBF accurately and noninvasively.

CEUS-based renal perfusion parameters have been documented in healthy animals. Time-dependent intensity curves can readily be generated based on selected regions of interest (ROI) in the renal cortex and medulla, as exemplified in Figure 1. Peak intensity (PI), time to peak intensity (TTP), mean transit time (MT'T), and area under the curve (AUC) are some of the key observational parameters that are typically used in renal perfusion studies, as illustrated in Figure 1. Different animals exhibited similar

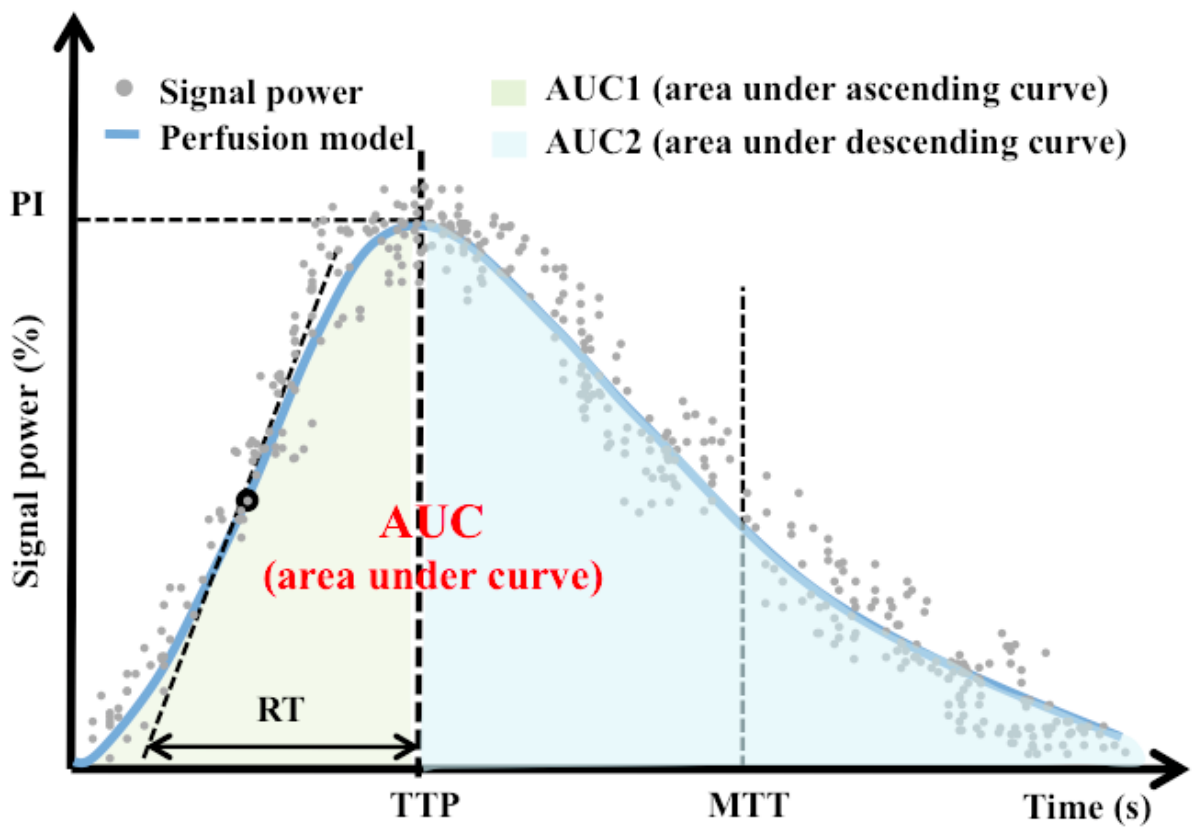

Figure 1: Contrast-enhanced ultrasound parameters derived from the perfusion model. These parameters include peak intensity (PI, with respect to PI of the reference ROI), rise time (RT), time to peak (TTP), and mean transit time (MTT). AUC (area under curve) is divided into two parts, which includes AUC1 (area under ascending curve) and AUC2 (area under descending curve) 
renal perfusion enhancement patterns under CEUS: the renal cortex enhanced initially followed by medullary enhancement ${ }^{[20-23]}$. Moreover, another study revealed that the location and size of ROIs used for this imaging approach did not make significant differences to the renal perfusion metrics captured using CEUS ${ }^{[21]}$. This result augurs well for the routine analysis of CEUS data based on ROIs in clinical practice.

CEUS has also been used for renal perfusion evaluation in pathological conditions. In diabetic Goto-Kakizaki rats, renal perfusion, as estimated by CEUS, consistently matched the pathological changes in the kidneys ${ }^{[24]}$. When compared to normal controls, dogs with iatrogenic hypercortisolism had significantly increased CEUS PI and AUC values in their kidneys compared to controls ${ }^{[25]}$. CEUS has also been successfully used for monitoring renal microvascular perfusion in ischemia/reperfusion injury in mice ${ }^{[26]}$, chronic renal ischemic injury in animal models ${ }^{[27]}$, porcine intra-abdominal hypertension ${ }^{[28]}$, as well as global hypoxia in piglets ${ }^{[29]}$. Examination of norepinephrinetreated dogs showed that CEUS parameters were predictive of effective renal plasma flow, suggesting that CEUS could also be used in assessing renal perfusion following pharmacologic intervention ${ }^{[30]}$.

Collectively, these studies in diverse animal models reveal that CEUS could be used for the non-invasive evaluation of renal microvascular perfusion under different disease settings. These animal studies have laid the foundation for further systematic evaluation of CEUS in human renal diatheses.

\section{RENAL MICROVASCULAR PERFUSION EVALUATION IN HUMANS USING CEUS}

Measurement of RBF in a clinical setting is highly challenging. Current methods include utilizing paraaminohippurate $(\mathrm{PAH})$ or a radioisotope tracer. Although PAH still remains a gold standard, the technical difficulties associated with its use have limited its routine application in the clinic, although it is commonly used for research. Nuclear medicine based techniques also pose problems such as requiring time for excretion of the injected radioisotopes out of the body, and concerns regarding radioisotope exposure. Against this backdrop, CEUS offers a novel approach for RBF evaluation, without the abovementioned concerns, as it appears to be fast, noninvasive, and safe. Observations in healthy volunteers reveal that CEUS-ascertained parameters exhibit good correlation with findings obtained using PAH ${ }^{[31]}$. CEUS has also been used to evaluate renal microvascular perfusion following the administration of vasoactive drugs. CEUS ascertained parameters decreased after the use of angiotensin II (Ang II), and increased after captopril (Ang II inhibitor) administration $^{[32]}$. Noradrenaline- ${ }^{[33]}$ and dopamineinduced ${ }^{[34]}$ changes in renal perfusion were also correctly validated by CEUS. Another CEUS-based study ${ }^{[35]}$ showed that diclofenac sodium decreased renal perfusion, but etodolac did not. Collectively, these studies justify the use of CEUS for monitoring the impact of novel drugs on renal perfusion.

In humans, CEUS has already been applied to the study of various kidney diseases, including chronic kidney disease $(\mathrm{CKD})^{[36]}$ (notably DKD ${ }^{[1,37]}$ ), AKI ${ }^{[38]}$, and kidney transplantation ${ }^{[39]}$. All of these investigations indicate that CEUS is well-tolerated, and that the subjects experience no side effects due to the contrast agents.

Studies document that CEUS could reflect renal perfusion in real-time in AKI ${ }^{[40]}$, and could predict acute rejection ${ }^{[41]}$ and delayed graft function ${ }^{[42]}$ in kidney transplantation. With progression of CKD stages, CEUS-ascertained renal enhancement showed delayed increase and decrease of PI, and acceleration of decay ${ }^{[43]}$. Quantitative analysis established that early stages of CKD (stages 1 and 2) were marked by decreased AUC compared to healthy controls ${ }^{[36]}$. Similar results were also reported in DKD ${ }^{[1,44]}$. By evaluating renal microvascular perfusion, CEUS could not only estimate renal dysfunction, but also could predict early kidney injury. Urinary microalbumin/creatinine (MALB/ UCR) is a well-known early biomarker for DKD, and its levels could increase before renal dysfunction. Ling et al. reportedthat in early stage DKD (CKD stage 1), patients with lower levels of urinary MALB/UCR ratio $(<10 \mathrm{~g} / \mathrm{mol})$ had decreased PI and acceleration of signal recession, when compared to patients with higher MALB/ $\mathrm{UCR}^{[1]}$, and similar findings are presented in Figure 2, for illustration purposes. Quantitative results showed that the AUC values could differentiate patients with different levels of $\mathrm{MALB} / \mathrm{UCR}^{[1]}$, indicating that CEUS could be utilized for early detection of DKD. Collectively, the above findings indicate that CEUS offers great potential in monitoring and predicting renal injury in acute and chronic renal diseases.

\section{LIMITATIONS AND FURTHER STUDIES}

The limitations of using CEUS to assess renal microvascular perfusion include the following. First, there is no international standard for evaluating renal microvascular perfusion; well-powered clinical trials are still needed to establish the parameters that are optimal for clinical evaluation and the normal range in different subjects groups. Second, the logistics associated with CEUS need to be factored in. Additional time may be needed for 


\section{CEUS Enhanced images in early stage of DKD}

$\mathrm{MALB} / \mathrm{UCR}$

$<10 \mathrm{~g} / \mathrm{mol}$
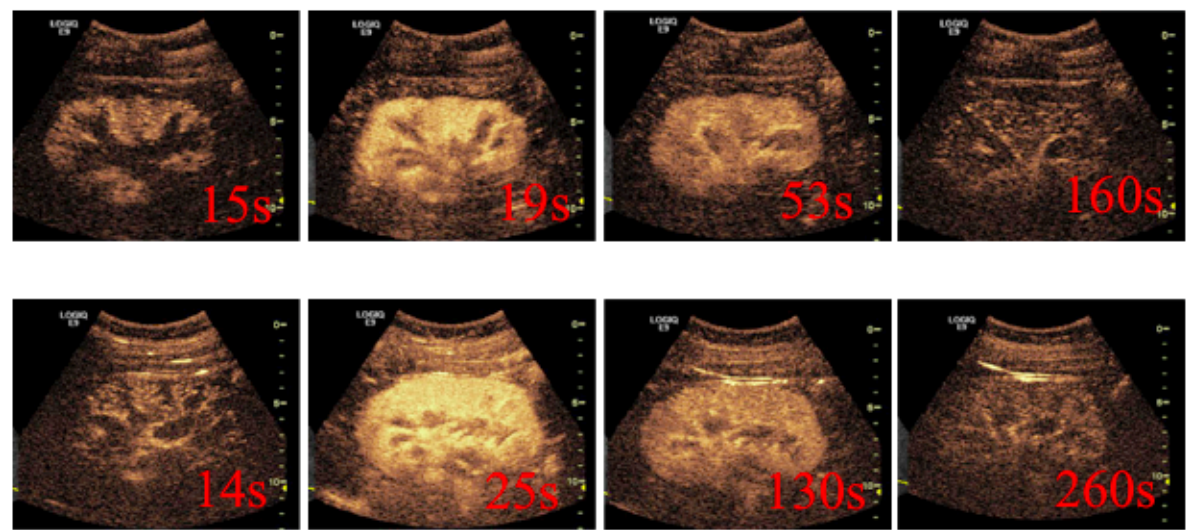

Figure 2: Representative serial contrast-enhanced images in early stage of diabetic kidney disease (DKD) patients with different levels of urinary microalbumin/creatinine ratio (MALB/UCR). Early stage of DKD is defined as DKD patients with estimated glomerular filtration rate $\geq 90 \mathrm{~mL} / \mathrm{min} / 1.73 \mathrm{~m}^{2}$ (chronic kidney disease stage 1 ). All subjects went through the same enhance and fading stages. Early stage of DKD patients with MALB/UCR $\geq 10 \mathrm{~g} / \mathrm{mol}$ got a higher peak intensity and increased time for

placing an intravenous catheter and quantitative analysis, while the cost of agents should also be considered. Third, patients with serious cardiac problems and pulmonary disease cannot be subjected to CEUS. However, this limitation may be less frequent than the contraindication for contrast enhanced computed tomography (CT) or magnetic resonance (MR) relating to patients' anaphylactic history or renal status. Finally, contrast agents used in CEUS are not excreted into the collecting system; thus, the latter will not be adequately imaged.

Despite these limitations, CEUS is still a promising method for renal microvascular evaluation. Well-designed and largescaled clinic trials that systematically evaluate the predictive power of CEUS in various settings of AKI and CKD are clearly needed. Looking to the future, it is exciting to note that the technology used for CEUS can potentially lend itself to ultrasound-directed therapeutic delivery systems using microbubbles. Given its diagnostic and potential therapeutic potential, CEUS appears likely to grow in prominence over the coming decade.

\section{Source of Foundation}

This article is supported by grants from the National Natural Science Foundation of China (81301626, 81571574) and the National Global Experts Recruitment Program of China (WQ20133100165).

\section{Conflict of Interest}

None declared.

\section{REFERENCES}

1. Wang L, Wu J, Cheng JF, Liu XY, Ma F, Guo LH, et al. Diagnostic value of quantitative contrast-enhanced ultrasound (CEUS) for early detection of renal hyperperfusion in diabetic kidney disease. J Nephrol 2015;28:66978.

2. Honore PM, Jacobs R, De Waele E, Diltoer M, Spapen HD. Renal blood flow and acute kidney injury in septic shock: an arduous conflict that smolders intrarenally? Kidney Int 2016;90:22-4.

3. Prowle JR, Molan MP, Hornsey E, Bellomo R. Measurement of renal blood flow by phase-contrast magnetic resonance imaging during septic acute kidney injury: a pilot investigation. Crit Care Med 2012;40:1768-76.

4. Rossi C, Artunc F, Martirosian P, Schlemmer HP, Schick F, Boss A. Histogram analysis of renal arterial spin labeling perfusion data reveals differences between volunteers and patients with mild chronic kidney disease. Invest Radiol 2012;47:490-6.

5. Daghini E, Primak AN, Chade AR, Krier JD, Zhu XY, Ritman EL, et al. Assessment of renal hemodynamics and function in pigs with 64-section multidetector CT: comparison with electron-beam CT. Radiology 2007;243:405-12.

6. Dujardin M, Sourbron S, Luypaert R, Verbeelen D, Stadnik T. Quantification of renal perfusion and function on a voxel-by-voxel basis: a feasibility study. Magn Reson Med 2005;54:841-9.

7. Michaely HJ, Kramer H, Oesingmann N, Lodemann KP, Reiser MF, Schoenberg SO. Semiquantitative assessment of first-pass renal perfusion at $1.5 \mathrm{~T}$ : comparison of $2 \mathrm{D}$ saturation recovery sequences with and without parallel imaging. AJR Am J Roentgenol 2007;188:919-26.

8. Marckmann P, Skov L, Rossen K, Dupont A, Damholt MB, Heaf JG, et al. Nephrogenic systemic fibrosis: suspected causative role of gadodiamide used for contrast-enhanced magnetic resonance imaging. J Am Soc Nephrol 2006;17:2359-62.

9. Wentland AL, Artz NS, Fain SB, Grist TM, Djamali A, Sadowski EA. MR measures of renal perfusion, oxygen bioavailability and total renal blood flow in a porcine model: noninvasive regional assessment of renal function. Nephrol Dial Transplant 2012;27:128-35.

10. Zhou HY, Chen TW, Zhang XM. Functional Magnetic Resonance Imaging in Acute Kidney Injury: Present Status. Biomed Res Int. 2016;2016:2027370.

11. Xu HX, Lu MD. The current status of contrast-enhanced ultrasound in 
China. J Med Ultrason (2001) 2010;37:97-106.

12. McArthur C, Baxter GM. Current and potential renal applications of contrast-enhanced ultrasound. Clin Radiol 2012;67:909-22.

13. Piscaglia F, Nolsoe C, Dietrich CF, Cosgrove DO, Gilja OH, Bachmann Nielsen $\mathrm{M}$, et al. The EFSUMB Guidelines and Recommendations on the Clinical Practice of Contrast Enhanced Ultrasound (CEUS): update 2011 on non-hepatic applications. Ultraschall Med 2012;33:33-59.

14. Cokkinos DD, Antypa EG, Skilakaki M, Kriketou D, Tavernaraki E, Piperopoulos PN. Contrast enhanced ultrasound of the kidneys: what is it capable of? Biomed Res Int 2013;2013:595873.

15. Tamai H, Takiguchi Y, Oka M, Shingaki N, Enomoto S, Shiraki T, et al. Contrast-enhanced ultrasonography in the diagnosis of solid renal tumors. J Ultrasound Med 2005;24:1635-40.

16. Correas JM, Claudon M, Tranquart F, Helenon AO. The kidney: imaging with microbubble contrast agents. Ultrasound Q 2006;22:53-66.

17. Cokkinos DD, Antypa E, Kalogeropoulos I, Tomais D, Ismailos E, Matsiras I, et al. Contrast-enhanced ultrasound performed under urgent conditions. Indications, review of the technique, clinical examples and limitations. Insights Imaging 2013;4:185-98.

18. Jimenez C, de Gracia R, Aguilera A, Alonso S, Cirugeda A, Benito J, et al. In situ kidney insonation with microbubble contrast agents does not cause renal tissue damage in a porcine model. J Ultrasound Med 2008;27:1607-15.

19. Kogan P, Johnson KA, Feingold S, Garrett N, Guracar I, Arendshorst WJ, et al. Validation of dynamic contrast-enhanced ultrasound in rodent kidneys as an absolute quantitative method for measuring blood perfusion. Ultrasound Med Biol 2011;37:900-8.

20. Schweiger H, Ohlerth S, Gerber B. Contrast-enhanced ultrasound of both kidneys in healthy, non-anaesthetized cats. Acta Vet Scand 2015;57:80.

21. Macri F, Di Pietro S, Liotta L, Piccionello AP, Pugliese M, De Majo M. Effects of size and location of regions of interest examined by use of contrast-enhanced ultrasonography on renal perfusion variables of dogs. Am J Vet Res 2016;77:869-76.

22. Yi K, Ji S, Kim J, Yoon J, Choi M. Contrast-enhanced ultrasound analysis of renal perfusion in normal micropigs. J Vet Sci 2012;13:311-4.

23. Mahoney M, Sorace A, Warram J, Samuel S, Hoyt K. Volumetric contrastenhanced ultrasound imaging of renal perfusion. J Ultrasound Med 2014;33:1427-37.

24. Ma F, Yadav GP, Cang YQ, Dang YY, Wang CQ, Liu B, et al. Contrastenhanced ultrasonography is a valid technique for the assessment of renal microvascular perfusion dysfunction in diabetic Goto-Kakizaki rats. Nephrology (Carlton) 2013;18:750-60.

25. Haers H, Daminet S, Smets PM, Duchateau L, Aresu L, Saunders JH. Use of quantitative contrast-enhanced ultrasonography to detect diffuse renal changes in Beagles with iatrogenic hypercortisolism. Am J Vet Res 2013;74:70-7.

26. Fischer K, Meral FC, Zhang Y, Vangel MG, Jolesz FA, Ichimura T, et al. High-resolution renal perfusion mapping using contrast-enhanced ultrasonography in ischemia-reperfusion injury monitors changes in renal microperfusion. Kidney Int 2016;89:1388-98.

27. Dong Y, Wang W, Cao J, Fan P, Lin X. Quantitative evaluation of contrastenhanced ultrasonography in the diagnosis of chronic ischemic renal disease in a dog model. PLoS One 2013;8:e70337.

28. Sui F, Zheng Y, Li WX, Zhou JL. Renal circulation and microcirculation during intra-abdominal hypertension in a porcine model. Eur Rev Med Pharmacol Sci 2016;20:452-61.

29. Brabrand K, de Lange C, Emblem KE, Reinholt FP, Saugstad OD, Stokke $\mathrm{ES}$, et al. Contrast-enhanced ultrasound identifies reduced overall and regional renal perfusion during global hypoxia in piglets. Invest Radiol 2014;49:540-6.

30. Arger PH, Sehgal CM, Pugh CR, Kirchoffer JI, Kotlar EY, Bovee KC. Evaluation of change in blood flow by contrast-enhanced power Doppler imaging during norepinephrine-induced renal vasoconstriction. J Ultrasound Med 1999;18:843-51.

31. Kalantarinia K, Belcik JT, Patrie JT, Wei K. Real-time measurement of renal blood flow in healthy subjects using contrast-enhanced ultrasound. Am J Physiol Renal Physiol 2009;297:F1129-34.

32. Schneider AG, Hofmann L, Wuerzner G, Glatz N, Maillard M, Meuwly $\mathrm{JY}$, et al. Renal perfusion evaluation with contrast-enhanced ultrasonography. Nephrol Dial Transplant 2012;27:674-81.

33. Schneider AG, Goodwin MD, Schelleman A, Bailey M, Johnson L, Bellomo R. Contrast-enhanced ultrasonography to evaluate changes in renal cortical microcirculation induced by noradrenaline: a pilot study. Crit Care 2014;18:653.

34. Kishimoto N, Mori Y, Nishiue T, Shibasaki Y, Iba O, Nose A, et al. Renal blood flow measurement with contrast-enhanced harmonic ultrasonography: evaluation of dopamine-induced changes in renal cortical perfusion in humans. Clin Nephrol 2003;59:423-8.

35. Imamura H, Hata J, Iida A, Manabe N, Haruma K. Evaluating the effects of diclofenac sodium and etodolac on renal hemodynamics with contrast-enhanced ultrasonography: a pilot study. Eur J Clin Pharmacol 2013;69:161-5.

36. Dong Y, Wang WP, Cao J, Fan P, Lin X. Early assessment of chronic kidney dysfunction using contrast-enhanced ultrasound: a pilot study. Br J Radiol 2014;87:20140350.

37. Wang L, Cheng JF, Sun LP, Song YX, Guo LH, Xu JM, et al. Use of Contrast-Enhanced Ultrasound to Study Relationship between Serum Uric Acid and Renal Microvascular Perfusion in Diabetic Kidney Disease. Biomed Res Int 2015;2015:732317.

38. Kalantarinia K. Novel imaging techniques in acute kidney injury. Curr Drug Targets 2009;10:1184-9.

39. Wang X, Yu Z, Guo R, Yin H, Hu X. Assessment of postoperative perfusion with contrast-enhanced ultrasonography in kidney transplantation. Int J Clin Exp Med 2015;8:18399-405.

40. Schneider AG, Goodwin MD, Schelleman A, Bailey M, Johnson L, Bellomo R. Contrast-enhanced ultrasound to evaluate changes in renal cortical perfusion around cardiac surgery: a pilot study. Crit Care 2013;17:R138.

41. Jin Y, Yang C, Wu S, Zhou S, Ji Z, Zhu T, et al. A novel simple noninvasive index to predict renal transplant acute rejection by contrast-enhanced ultrasonography. Transplantation 2015;99:636-41.

42. Grzelak P, Szymczyk K, Strzelczyk J, Kurnatowska I, Sapieha M, Nowicki $\mathrm{M}$, et al. Perfusion of kidney graft pyramids and cortex in contrastenhanced ultrasonography in the determination of the cause of delayed graft function. Ann Transplant 2011;16:48-53.

43. Tsuruoka K, Yasuda T, Koitabashi K, Yazawa M, Shimazaki M, Sakurada $\mathrm{T}$, et al. Evaluation of renal microcirculation by contrast-enhanced ultrasound with Sonazoid as a contrast agent. Int Heart J 2010;51:176-82.

44. Dong Y, Wang WP, Lin P, Fan P, Mao F. Assessment of renal perfusion with contrast-enhanced ultrasound: Preliminary results in early diabetic nephropathies. Clin Hemorheol Microcirc 2015;62:229-38.

How to cite this article: Wang L, Mohan C. Contrast-enhanced ultrasound: A promising method for renal microvascular erfusion evaluation. J Transl Intern Med 2016; 4: 104-8. 